\title{
Electron Probes for Ultrafast Dynamics at the Nanoscale
}

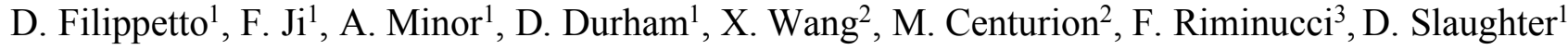 \\ 1. Lawrence Berkeley National Laboratory, Berkeley, California, USA \\ 2. Department of Physics and Astronomy, University of Nebraska-Lincoln, Lincoln, Nebraska, USA \\ 3. University of Rome "Sapienza", Rome, Italy
}

Ultrafast electron diffraction (UED) and microscopy (UEM) have seen a rapidly increasing interest in the last years due to the availability of high-field electron sources capable of producing femtosecond pulses. Thanks to the use of technology and techniques inherited from particle accelerators and Free Electron Lasers, high-density electron pulses are generated via photoemission and promptly accelerated to relativistic energies, minimizing the detrimental effect of repulsive electrostatic forces and electronelectron scattering. Rapidly oscillating fields in the radiofrequency (RF) range are used to boost the accelerating field at photoemission in the tens of Megavolt/meter (MV/m), thus packing millions of electrons in femtosecond bursts [1][2]. On the other hand, such high peak current is generated via photoemission from large areas, in detriment of the transverse beam brightness and, therefore, spatial resolution. A typical UED experiment is averaging sample response over micrometer/millimiter-scale areas, loosing information on the local dynamics. Also, the average dose delivered at the sample is orders of magnitude smaller than typical Transmission Electron Microscopes, making it hard to study complex systems in low-density targets such as gases and liquids, or isolated meso-scale objects requiring large lateral coherence length.

At Lawrence Berkeley National Laboratory we are developing a new UED beamline (HiRES) [3]. It makes use of a unique electron source [4] to deliver at the sample up to $10^{12}$ electrons per second with femtosecond resolution, an increase of 3 orders of magnitude over state-of-art MeV UED setups. The electron gun produces picosecond-long pulses at $750 \mathrm{keV}$ kinetic energy, with up to $10^{6}$ electrons at a repetition rate of $1 \mathrm{MHz}$. A subsequent RF cavity acts as a longitudinal focusing lens, shrinking the pulse duration down to femtoseconds. Along the beamline, the pulse is shaped for optimal spatial and temporal resolution via the use of transversely focusing lenses, dispersive elements and collimators, allowing a large flexibility in the parameter space. The project aims at broadening the scientific reach of UED setups by enabling ultrafast nano-diffraction and Scanning Transmission Microscopy on one side, and extending the experimental capabilities of gas-phase UED to complex multi-molecule aggregates.

First experiments have been conducted on producing picometer-scale emittance beams for nanofocusing on solid state samples. Sub-micrometer dimensions are generated with the use of high-gradient in-vacuum focusing lenses with $2.5 \mathrm{~cm}$ focal length. Scanning of the nano-beam over the sample reveals information on the behaviour of different grains in the samples, and demonstrates the capability of performing Ultrafast STEM.

The experimental chamber has lately been modified to include a nozzle for injection of gas-phase targets. Good signal-to-noise ration on molecular scattering signal was achieved on calibration molecules like $\mathrm{N}_{2}$ and $\mathrm{CO}_{2}$, with integration times shorter than 1 second.

The high flux of the HiRES source can also be used to increase the lateral coherence of the beam. While large coherence lengths can be obtained by collimation and beam size manipulation [4], the most 
effective technique is by physically limiting the emission area to sub-micrometer dimensions [5]. The consequent decrease of electron flux is then compensated by the $\mathrm{MHz}$ repetition repetition rate of the instrument. RF-based electron sources require flat surfaces for electron beam generation, as the use of tips is inhibited by emitter stability and field-emission in the strong accelerating field environment. We are exploring the use of surface-plasmon-polariton (SPP) interference, with the aim of decreasing the source size by more than 2 orders of magnitude. We were able to fabricate nanostructures achieving large field enhancement on flat surfaces, with the SPP field pointing longitudinally, parallel to the externally applied accelerating field, obtaining small electron sources with small angular spread. The latest results on this topic from our laboratory promise to increase the lateral coherence of the beam by at least two orders of magnitude.

\section{References:}

[1] D Filippetto, H Qian, Journal of Physics B: Atomic, Molecular and Optical Physics 49, p. 104003. [2] SP Weathersby et al, Review of Scientific Instruments 86, p. 073702.

[3] F Sannibale et al, Physical Review Special Topics-Accelerators and Beams 15, p. 103501.

[4] D Filippetto, H Qian, Journal of Physics B: Atomic, Molecular and Optical Physics 49, p. 104003

[5] D Filippetto et al, Physical Review Special Topics-Accelerators and Beams 17, p. 024201.

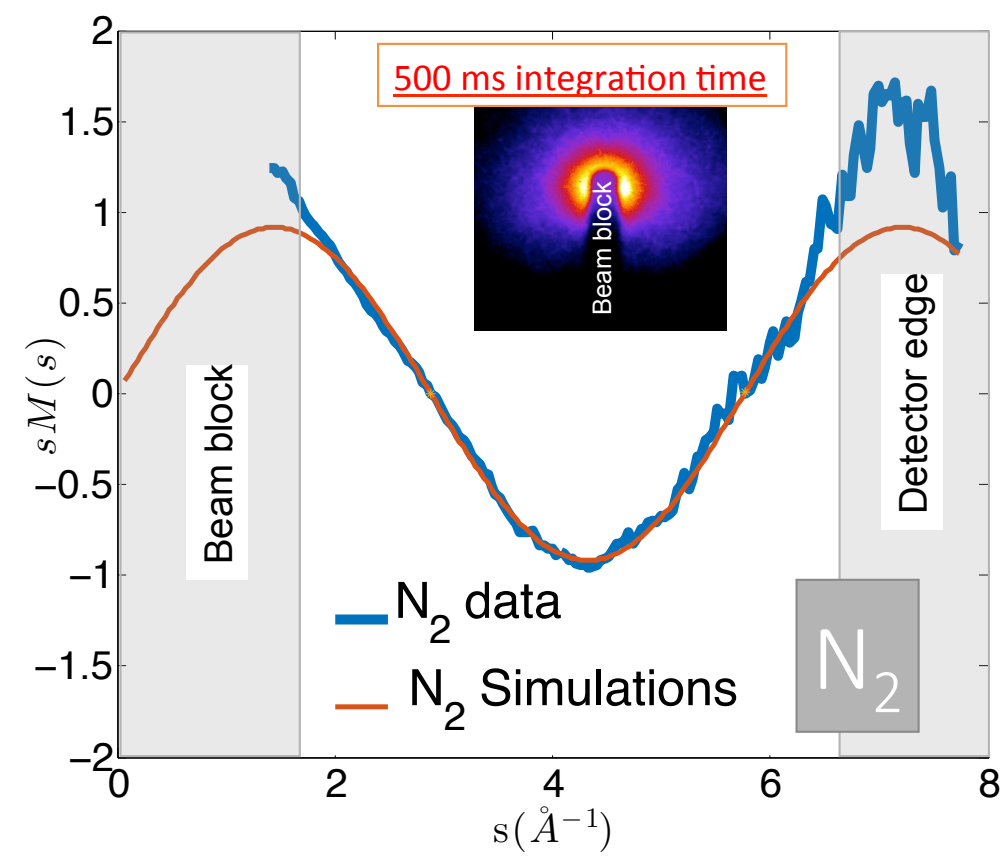

Figure 1. Example of gas-phase diffraction data from $\mathrm{N}_{2}$. The signal was taken by integrating only 500 milliseconds. The gas nozzle has an aperture of $100 \mu \mathrm{m}$ and the gas pressure right before the nozzle was 800 Torr. 